\title{
A New Approach to Constructing Optimal Prefix Circuits with Small Depth
}

\author{
Yen-Chun Lin* and Jun-Wei Hsiao** \\ *Dept. of Computer Science and Information Engineering \\ **Dept. of Electronic Engineering \\ National Taiwan University of Science and Technology, Taipei 106, Taiwan \\ E-mail: yclin@computer.org
}

\begin{abstract}
Prefix computation has many applications, and should be implemented as a primitive operation. Many combinational circuits for performing the prefix operation in parallel, called parallel prefix circuits, have been designed and studied. The size of a prefix circuit $D, s(D)$, is the number of operation nodes in $D$, and the depth of $D, d(D)$, is the maximum level of operation nodes in D. Smaller depth implies faster computation, while smaller size implies less power consumption and smaller area in VLSI implementation and thus less cost. $D$ is depth-size optimal if $d(D)+$ $s(D)=2 n-2$. Another circuit parameter is fan-out. A circuit having a smaller fan-out is faster and smaller in VLSI implementation. Thus, a circuit should have a small fan-out for it to be of practical use. In this paper, we take a new approach to designing a depth-size optimal parallel prefix circuit, WE4, with fan-out 4 and small depth. In many cases of n, WE4 has the smallest depth among all known prefix circuits.
\end{abstract}

\section{Introduction}

Given $n$ values $x_{1}, x_{2}, \ldots, x_{n}$ and an associative binary operation $\otimes$, the prefix operation is to compute the $n$ values $x_{1} \otimes x_{2} \otimes \ldots \otimes x_{i}, 1 \leq i \leq n$. Prefix computation has many applications, such as sorting, job scheduling, loop parallelization, and processor allocation [1, 2, 7-9, 17]. Because of its usefulness, it should be implemented as a primitive operation [3]. Many combinational circuits for performing the prefix operation in parallel, called parallel prefix circuits, have been designed and studied [2, 4, 5, 8-11, 13-16].

In this paper, we assume that the number of inputs is $n$, unless otherwise stated. We represent a prefix circuit with a directed acyclic graph containing $n$ input nodes, $n$ output nodes, at least $n-1$ operation nodes, and at least one duplication node. For ease of presentation, in this paper, all the directed edges are assumed to be downward; thus, the arrows need not be shown. A serial prefix circuit $S(n)$ is shown in Fig. 1 , in which vertical edges from left to right are named line 1 , line $2, \ldots$, line $n$, respectively. Input nodes are on the top of a circuit and have indegree 0 and outdegree 1, representing input items. Output nodes are at the bottom and have indegree 1 and outdegree 0 , representing outputs. An operation node, represented by a black dot, performs the $\otimes$ operation on its two inputs, having indegree 2 and outdegree 1 or more. For any operation node on line $i$ at level $j$, its left input is from a node at level $j-1$, while its right input is always from line $i$. A duplication node has indegree 1 and outdegree 2 or more; it outputs multiple copies of its single input. In Fig. 1, a duplication node is on line 1 at level 0 . The numbers at the left of a prefix circuit denote the depth levels of the nodes to the right. The input node on line $i$ represents input $x_{i}$. When no confusion is caused, we may use simply $D$ for any prefix circuit $D(p)$, where $p$ may be the number of inputs or a parameter with another meaning.

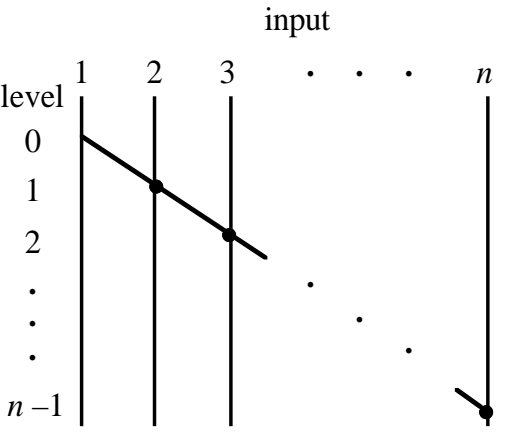

Fig. 1. Serial prefix circuit $S(n)$.

The size of a prefix circuit $D, s(D)$, is the number of operation nodes in $D$, and the depth of $D$, $d(D)$, is the maximum level of operation nodes in $D$. Smaller depth implies faster computation. Smaller size implies less power consumption and smaller area in VLSI implementation and thus less cost. Therefore, size and depth are important parameters of prefix circuits. For any prefix circuit $\mathrm{D}, d(D)+s(D) \geq$ $2 n-2$ [16]. Thus, $D$ is depth-size optimal, or optimal for short, if $d(D)+s(D)=2 n-2$. For 
example, Fig. 1 shows that $s(S)=d(S)=n-1$; thus, $S$ is optimal.

A third important parameter is fan-out. The fan-out of a node is its outdegree. A node having a smaller fan-out is faster and smaller in VLSI implementation [18]. A node has unbounded fan-out if the fan-out is not fixed and is a function of $n$; otherwise, the fan-out of the node is a constant, or is bounded. The fan-out of prefix circuit $D, f o(D)$, is the maximum fan-out of all nodes in $D$. For example, $f o(S)=2$. A circuit has unbounded fan-out if one of its nodes has unbounded fan-out. A circuit should have a small bounded fan-out for it to be of practical use.

For ease of presentation, let $i: j$ represent the result of computing $x_{i} \otimes x_{i+1} \otimes \ldots \otimes x_{j}$, where $i \leq j$. We use $i a(D)=a$ to denote that line 1 of prefix circuit $D$ has a duplication node at level $a$ and has no duplication nodes at any level less than $a$. In addition, we use $l(D)=b$ to denote that line $n$ of $D$ obtains $1: n$ at level $b$.

Many previous parallel prefix circuits are briefly reviewed in [11]. Some of them with smaller depth are in the following. $L Y D$ is an optimal prefix circuit with unbounded fan-out, whose depth is between $2\lceil\lg n\rceil-6$ and $2\lceil\lg n\rceil-3[9] . \quad M$ is also an optimal prefix circuit with unbounded fan-out, whose depth is between $2\lceil\lg n\rceil-5$ and $2\lceil\lg n\rceil-3$ [12]. $\mathrm{H} 4$ is an optimal prefix circuit with fan-out 4, whose depth is between $2\lceil\lg n\rceil-5$ and $2\lceil\lg n\rceil-3[11]$. $Z 4$ is an optimal prefix circuit with fan-out 4 , whose depth is between $2\lceil\lg n\rceil-6$ and $2\lceil\lg n\rceil-3[6]$.

In this paper, we introduce a new optimal parallel prefix circuit, denoted as $W E 4$, with fan-out 4 . In many cases of $n$, it has the smallest depth among all known prefix circuits. Section 2 briefly reviews previous results. Sections 3 and 4 present prefix circuits $W L$ and $W V$, respectively; these circuits will be used in Section 5 to construct WE4. Section 6 compares WE4 with the optimal parallel prefix circuits mentioned in the previous paragraph in some detail. Section 7 concludes this paper.

\section{Brief review of previous results}

A prefix circuit $D$ can be defined with sets of operation nodes at level $i, i=1,2, \ldots, d(D)$ [16]:

$G_{i}=\{(x, y) \mid$ at level $i$ on line $y$ there is an operation node whose left input is the output of a node on line $x$ at level $i-1\}$.

If $(x, y) \in G_{i}$, the corresponding operation node can be denoted as $(x, y)_{i}$.

\subsection{Prefix circuits $Q$ and $W$}

Prefix circuit $Q(n)$ with fan-out at most 4 has been presented in [14].

Property 1 [14]: The depth of $Q(n)$ is

$$
\begin{array}{ll}
\lceil\lg n\rceil & \text { if } n \leq 7, \\
2 t-4 & \text { if } t \geq 4 \text { and } 2^{t-1} \leq n<3 \times 2^{t-2}, \\
2 t-3 & \text { if } t \geq 4 \text { and } 3 \times 2^{t-2} \leq n<2^{t} .
\end{array}
$$

$W(n)$ is a prefix circuit defined with the following operation nodes [11]:

$$
\begin{aligned}
& G_{i}=\{(i+1, i+2)\}, \quad i=1,2, \ldots, n-2 ; \\
& G_{n-1}=\{(1, i) \mid i=2,3, \ldots, n\} .
\end{aligned}
$$

For example, $W(3)$ and $W(4)$ are shown in Fig. 2. By definition, $d(W)=n-1, s(W)=2 n-3$, $i a(W)=n-2$, and $l(W)=n-1$.
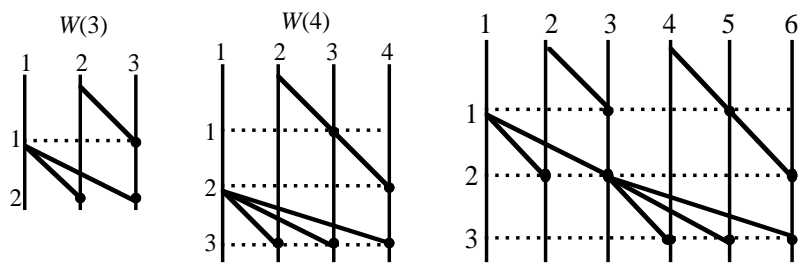

Fig. 2. $W(3), W(4)$, and $W(3) \cdot W(4)$.

\subsection{Composition of prefix circuits, size optimality, and other properties}

Assume that $A\left(n_{1}\right)$ and $B\left(n_{2}\right)$ are two prefix circuits with $n_{1}$ and $n_{2}$ inputs, respectively. $A\left(n_{1}\right)$ and $B\left(n_{2}\right)$ can be composed into a prefix circuit with $n_{1}+n_{2}-1$ inputs by merging the operation node that produces $1: n_{1}$ on line $n_{1}$ of $A\left(n_{1}\right)$ with the first, if not unique, duplication node on line 1 of $B\left(n_{2}\right)$; the resulting circuit is denoted by $A\left(n_{1}\right) \cdot B\left(n_{2}\right)$ [16]. For example, Fig. 2 also gives $W(3) \bullet W(4)$. Three or more prefix circuits can also be composed into a single prefix circuit, and the composition operation is associative.

Definition 2 [11]: For any prefix circuit $A$ with $n$ inputs, if $i a(A)=a, l(A)=b$, and $s(A)=2 n-2$ $+a-b$, then $A$ is size optimal; we say that $A$ is $\operatorname{SOPC}(n, a, b)$.

Property 3 [11]: $Q(n)$ is $\operatorname{SOPC}(n, 0,\lceil\lg n\rceil)$.

Property 4 [11]: $W(n)$ is $\operatorname{SOPC}(n, n-2, n-$ 1) with fan-out $n$.

Property 5 [11]: If $A$ is $\operatorname{SOPC}(n, 0, b)$ and $d(A)=b$, then $A$ is optimal.

Property 6 [11]: If $A\left(n_{1}\right)$ and $B\left(n_{2}\right)$ are $\operatorname{SOPC}\left(n_{1}, a, b\right)$ and $S O P C\left(n_{2}, c, d\right)$, respectively, where $b \geq c$, then $A\left(n_{1}\right) \cdot B\left(n_{2}\right)$ is 
$\operatorname{SOPC}\left(n_{1}+n_{2}-1, a, b-c+d\right)$ with depth $\max \left\{d\left(A\left(n_{1}\right)\right), d\left(B\left(n_{2}\right)\right)+b-c\right\}$.

\section{Size optimal prefix circuit $W L$}

Prefix circuit $W L(5)$ is defined with the following operation nodes:

$$
\begin{aligned}
G_{1} & =\{(2,3),(6,7),(10,11)\}, \\
G_{2}= & \{(3,4),(7,8),(11,12)\}, \\
G_{3}= & \{(4,5),(8,9),(12,13)\}, \\
G_{4}= & (5,9)\}, \\
G_{5}= & \{(9,13)\}, \\
G_{6}= & \{(1,5),(1,9),(1,13)\}, \\
G_{7}=\{(1,2),(1,3),(1,4),(5,6),(5,7),(5,8), & (9,10),(9,11),(9,12)\} .
\end{aligned}
$$

Fig. 3 shows that $d(W L(5))=7, f o(W L(5))=4$, $i a(W L(5))=5$, and $l(W L(5))=6$. In addition, $s(W L(5))=23=2 \times 13-2+5-6$. Therefore, by Definition 2, $W L(5)$ is $\operatorname{SOPC}(13,5,6)$.

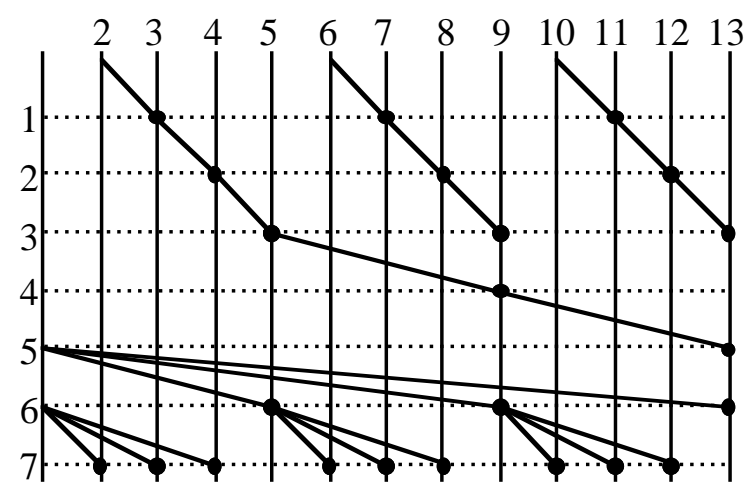

Fig. 3. $W L(5)$.

We can move the operation node $(1,13)_{6}$ of $W L(5)$ downward by 1 level to be $(1,13)_{7}$, and move the other operation nodes at level 6 and level 7 downward by 2 levels, resulting in a new prefix circuit $W A(5)$. Note that $d(W A(5))=9, f o(W A(5))=4$, and $W A(5)$ is $S O P C(13,6,7)$. We can also obtain $W B(5)$ by moving down all the nodes at levels 6 and 7 of $W L(5)$ by 2 levels. Clearly, $d(W B(5))=9, f o(W B(5))=4$, and $W B(5)$ is $\operatorname{SOPC}(13,7,8)$.

By Property 6, WA(5) $-W B(5)$ is $\operatorname{SOPC}(25,6,8)$. We can delete the operation node $(13,25)_{8}$ of $W A(5) \cdot$ $W B(5)$, and then add operation nodes $(13,25)_{6}$ and $(1,25)_{7}$, obtaining $W L(6)$ as shown in Fig. 4. Clearly, $d(W L(6))=9, f o(W L(6))=4$ and $i a(W L(6))=6$. Since only operation nodes on line 25 are modified and $W L(6)$ obtains $1: 25$ at level 7 , clearly $W L(6)$ is a prefix circuit and $l(W L(6))=7$.

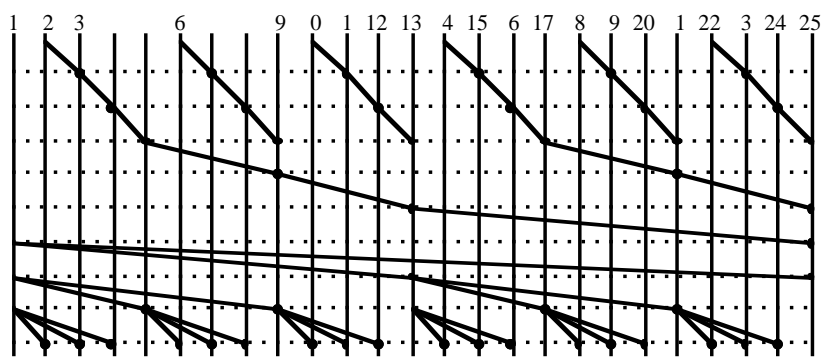

Fig. 4. $W L(6)$.

As already mentioned, $W A(5) \cdot W B(5)$ is $S O P C(25,6,8)$, thus $s(W A(5) \cdot W B(5))=2 \times 25-2$ $+6-8=46$. Clearly, $s(W L(6))=47$. That is, $s(W L(6))=2 \times 25-2+6-7$. Since $i a(W L(6))=6$ and $l(W L(6))=7$, by Definition 2, WL (6) is $\operatorname{SOPC}(25,6,7)$.

The above method of obtaining $W L(6)$ from $W L(5)$ can be generalized to derive $W L(t+1)$ from $W L(t)$, $t \geq 5$.

Algorithm A: Let $N$ be the set of nodes at levels $t+1$ through $2 t-3$ of $W L(t)$, where $t \geq 5$. We can obtain $W L(t+1)$ by the following procedures.

1. Move down the operation node $\left(1,3 \times 2^{t-3}+\right.$ $1)_{t+1}$ of $W L(t)$ by 1 level to become $\left(1,3 \times 2^{t-3}\right.$ $+1)_{t+2}$, and move down all the other operation nodes in $N$ by 2 levels, resulting in $W A(t)$. (If the left input of an operation node comes from a duplication node, then moving the operation node implies that the duplication node should also be moved accordingly.)

2. Move down all the operation nodes in $N$ by 2 levels to obtain $W B(t)$.

3. Delete node $\left(3 \times 2^{t-3}+1,3 \times 2^{t-2}+1\right)_{t+3}$ of the composed prefix circuit $W A(t) \cdot W B(t)$, and add 2 nodes $\left(3 \times 2^{t-3}+1,3 \times 2^{t-2}+1\right)_{t+1}$ and $\left(1,3 \times 2^{t-2}+1\right)_{t+2}$, resulting in $W L(t+1)$.

Theorem 7: $W L(t)$ is $\operatorname{SOPC}\left(3 \times 2^{t-3}+1, t, t+\right.$ 1) with depth $2 t-3$ and fan-out 4 , for $t \geq 5$.

Proof: This theorem can be proved by induction on $t$. The proof is omitted because of space limitation.

\section{Size optimal prefix circuit $W V$}

Prefix circuit $W V(5)$ is defined with the following operation nodes:

$$
\begin{aligned}
& G_{1}=\{(2,3),(5,6),(8,9)\}, \\
& G_{2}=\{(3,4),(6,7),(9,10)\}, \\
& G_{3}=\{(4,7)\}, \\
& G_{4}=\{(7,10)\}, \\
& G_{5}=\{(1,4),(1,7),(1,10)\},
\end{aligned}
$$


$G_{6}=\{(1,2),(1,3),(4,5),(4,6),(7,8),(7,9)\}$.

Fig. 5 shows that $d(W V(5))=6, f o(W V(5))=4$, $i a(W V(5))=4$, and $l(W V(5))=5$. In addition, $s(W V(5))=17=2 \times 10-2+4-5$. Therefore, by Definition 2, $W V(5)$ is $\operatorname{SOPC}(10,4,5)$.

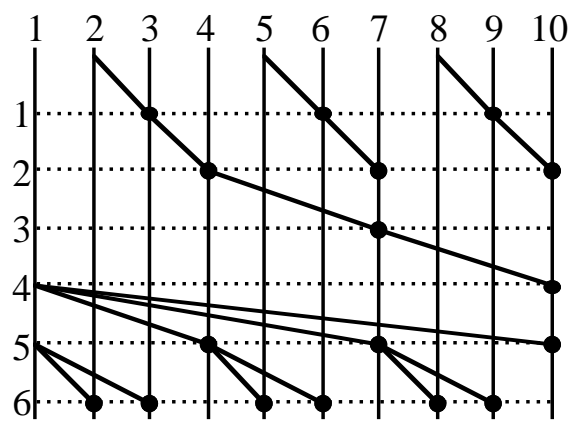

Fig. 5. $W V(5)$.

We can move down the operation node $(1,10)_{5}$ of $W V(5)$ by 1 level to be $(1,10)_{6}$, and move the other operation nodes at level 5 and level 6 downward by 2 levels, resulting in a new prefix circuit $V A(5)$. Note that $d(V A(5))=8, f o(V A(5))=3$, and $V A(5)$ is $S O P C(10,5,6)$. We can also obtain $V B(5)$ by moving down all the nodes at levels 5 and 6 of $W V(5)$ by 2 levels. Clearly, $d(V B(5))=8, f o(V B(5))=4$, and $V B(5)$ is $\operatorname{SOPC}(10,6,7)$.

By Property 6, $V A(5) \cdot V B(5)$ is $\operatorname{SOPC}(19,5,7)$. We can delete the operation node $(10,19)_{7}$ of $V A(5) \cdot$ $V B(5)$, and then add operation nodes $(10,19)_{5}$ and $(1,19)_{6}$, obtaining $W V(6)$ as shown in Fig. 6. Clearly, $d(W V(6))=8, f o(W V(6))=3$, and $i a(W V(6))=5$. Since only operation nodes on line 19 are modified and $W V(6)$ obtains $1: 19$ at level 6 , clearly $W V(6)$ is a prefix circuit and $l(W V(6))=6$.

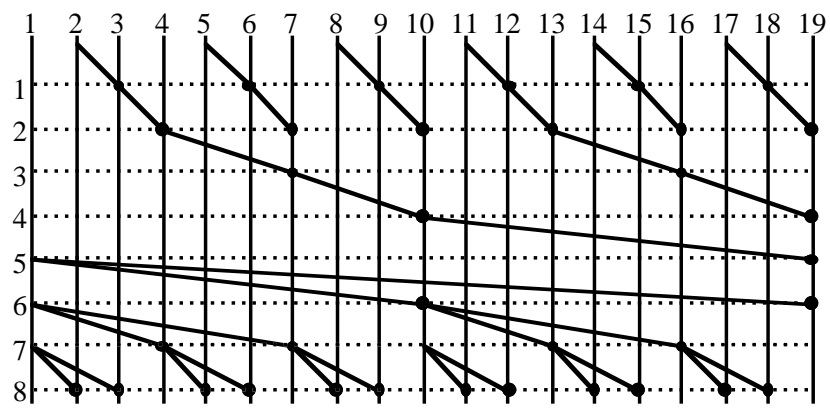

Fig. 6. $W V(6)$.

Since $V A(5) \cdot V B(5)$ is $\operatorname{SOPC}(19,5,7), s(V A(5)$ - $V B(5))=2 \times 19-2+5-7=34$, Thus, $s(W V(6))=$ $s(V A(5) \cdot V B(5))-1+2=2 \times 19-2+5-6$. Furthermore, since $i a(W V(6))=5$ and $l(W V(6))=6$, by Definition 2, $W V(6)$ is $\operatorname{SOPC}(19,5,6)$.

The above method of obtaining $W V(6)$ from $W V(5)$ can be generalized to derive $W V(t+1)$ from $W V(t)$, $t \geq 5$.

Algorithm B: Let $N$ be the set of nodes at levels $t$ through $2 t-4$ of $W V(t)$, where $t \geq 5$. We can obtain $W V(t+1)$ by the following procedures.

1. Move down the operation node $\left(1,9 \times 2^{t-5}+1\right)_{t}$ of $W V(t)$ by 1 level to become $\left(1,9 \times 2^{t-5}+1\right)_{t+1}$, and move down all the other operation nodes in $N$ by 2 levels, resulting in $V A(t)$.

2. Move down all the operation nodes in $N$ by 2 levels to obtain $V B(t)$.

3. Delete the node $\left(9 \times 2^{t-5}+1,9 \times 2^{t-4}+1\right)_{t+2}$ of the composed prefix circuit $V A(t) \cdot V B(t)$, and add operation nodes $\left(9 \times 2^{t-5}+1,9 \times 2^{t-4}+1\right)_{t}$ and $\left(1,9 \times 2^{t-4}+1\right)_{t+1}$, resulting in $W V(t+1)$.

Theorem 8: $W V(t)$ is $\operatorname{SOPC}\left(9 \times 2^{t-5}+1, t-1\right.$, $t$ ) with depth $2 t-4$ and fan-out 3 , for $t \geq 6$.

Proof: This theorem can be proved by induction on $t$. The proof is omitted because of space limitation.

\section{Depth-size optimal prefix circuit $W E 4$}

For ease of presentation, let $W G(t)=W L(t) \cdot$ $W L(t-1) \cdot \ldots \cdot W L(5) \cdot W(4)$, for $t \geq 5$, and $W G(4)=W(4)$.

Lemma 9: $W G(t)$ is $\operatorname{SOPC}\left(3 \times 2^{t-2}-8, t, 2 t-\right.$ 3 ) with depth $2 t-3$, for $t \geq 5$.

Proof: By Theorem 7, WL(t) is $\operatorname{SOPC}\left(3 \times 2^{t-3}+\right.$ $1, t, t+1)$ with depth $2 t-3$. By Property 6, $W L(t) \cdot W L(t-1)$ is $\operatorname{SOPC}\left(3 \times 2^{t-3}+3 \times 2^{t-4}+\right.$ $1, t, t+2)$ with depth $2 t-3$. Using Property 6 repeatedly, we can obtain that $W L(t) \cdot W L(t-1)$ -... WL(5) is $\operatorname{SOPC}\left(3 \times 2^{t-3}+3 \times 2^{t-4}+\ldots+3 \times 2^{2}\right.$ $+1, t, 2 t-4)$, i.e., $\operatorname{SOPC}\left(3 \times 2^{t-2}-11, t, 2 t-4\right)$, with depth $2 t-3$. Since $W(4)$ is $\operatorname{SOPC}(4,2,3)$ with depth 3 , by Property 6, $W G(t)=W L(t) \cdot W L(t-1)$ $\cdot \ldots \cdot W L(5) \cdot W(4)$ is $\operatorname{SOPC}\left(3 \times 2^{t-2}-8, t, 2 t-3\right)$ with depth $2 t-3$.

Q.E.D.

Assume that $n \geq 21$ and $t=\lceil\lg n\rceil$, we define $n$ input prefix circuit $W E 4(n)$ as follows:

1. When $21 \leq n \leq 28$ or $63 \leq n \leq 64$,

$$
\begin{gathered}
W E 4(n)=Q\left(n-21 \times 2^{t-5}+9\right) \cdot W V(t) \cdot \\
W G(t-1) .
\end{gathered}
$$

2. When $29 \leq n \leq 32$;

$$
\begin{gathered}
37 \times 2^{t-5}-9 \leq n \leq 5 \times 2^{t-2}-8, t \geq 6 \text {; or } \\
n=7 \times 2^{t-2}-9, t \geq 5, \\
W E 4(n)=Q\left(n-3 \times 2^{t-2}+8\right) \cdot S(2) \cdot W G(t) .
\end{gathered}
$$


3. When $48 \leq n \leq 62$;

$$
\begin{gathered}
5 \times 2^{t-3}-7 \leq n \leq 7 \times 2^{t-3}-10, t \geq 6 ; \\
7 \times 2^{t-3}-8 \leq n \leq 2^{t}, t \geq 7 ; \text { or } \\
2^{t}<n \leq 9 \times 2^{t-3}-10, t \geq 7, \\
W E 4(n)=Q\left(n-3 \times 2^{t-3}+9\right) \cdot W G(t-1) .
\end{gathered}
$$

4. When $9 \times 2^{t-4}-9 \leq n \leq 37 \times 2^{t-6}-10, t \geq 8$,

$$
W E 4(n)=Q\left(n-21 \times 2^{t-5}+9\right) \cdot W V(t-1)
$$$$
\text { - } W G(t-2) \text {. }
$$

As an example, Fig. 7 shows WE4(27).

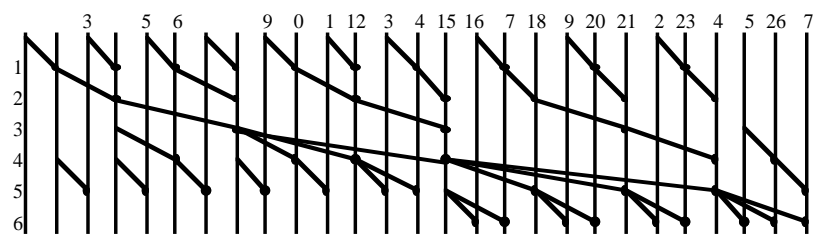

Fig. 7. $W E 4(27)=Q(15) \cdot W V(5) \cdot W(4)$.

Theorem 10: $W E 4(n)$ is an optimal prefix circuit with fan-out 4 and depth

$2\lceil\lg n\rceil-3 \quad$ when $29 \leq n \leq 32$;

$2\lceil\lg n\rceil-4$ when $21 \leq n \leq 28,47 \leq n \leq 64$, or $7 \times 2^{t-3}-9 \leq n \leq 2^{t}$, where $t \geq 7$;

$2\lceil\lg n\rceil-5$ when $33 \leq n \leq 46$ or $37 \times 2^{t-6}-9$ $\leq n \leq 7 \times 2^{t-3}-10$, where $t \geq 7$;

$2\lceil\lg n\rceil-6$ when $2^{t-1}<n \leq 37 \times 2^{t-6}-10$, where $t \geq 8$.

Proof: The proof is through distinguishing 10 cases. Because the processes for the cases each are very similar, we will give details for the first case only and give tips for the others.

Case 1: When $21 \leq n \leq 28$, let $m=n-12$; thus 9 $\leq m \leq 16$ and $\lceil\lg m\rceil=4$. By Property 1 , we have $4 \leq d(Q(m)) \leq 6$. By Property 3 , $Q(m)$ is $\operatorname{SOPC}(m, 0,4)$. As already mentioned, $W V(5)$ is $\operatorname{SOPC}(10,4,5)$ with depth 6. Thus, by Property 6, $Q(m) \cdot$ $W V(5)$ is $\operatorname{SOPC}(m+9,0,5)$. Since $W(4)$ is $\operatorname{SOPC}(4,2,3)$, by Property $6, W E 4(n)=$ $Q(m) \cdot W V(5) \cdot W(4)$ is $S O P C(m+12$, 0 ,6) with depth $6=2\lceil\lg n\rceil-4$. Finally, by Property $5, W E 4(n)$ is optimal.

Case 2: When $29 \leq n \leq 32$, let $m=n-16$.

Case 3: When $48 \leq n \leq 62$, let $m=n-15$.

Case 4: When $63 \leq n \leq 64$, let $m=n-33$.

Case 5: When $37 \times 2^{t-6}-9 \leq n \leq 5 \times 2^{t-3}-8$, where $t \geq 7$, let $m=n-3 \times 2^{t-3}+8$.

Case 6: When $5 \times 2^{t-3}-7 \leq n \leq 7 \times 2^{t-3}-10$, where $t \geq 6$, let $m=n-3 \times 2^{t-3}+9$.

Case 7: When $n=7 \times 2^{t-3}-9$, where $t \geq 6$, let $m$ $=n-3 \times 2^{t-3}+8$.
Case 8: When $7 \times 2^{t-3}-8 \leq n \leq 2^{t}$, where $t \geq 7$, let $m=n-3 \times 2^{t-3}+9$.

Case 9: When $2^{t-1}<n \leq 9 \times 2^{t-4}-10$, where $t \geq$ 8 , let $m=n-3 \times 2^{t-4}+9$.

Case 10: When $9 \times 2^{t-4}-9 \leq n \leq 37 \times 2^{t-6}-10$, where $t \geq 8$, let $m=n-21 \times 2^{t-6}+9$.

By Case 2 , when $29 \leq n \leq 32$, we have $d$ (WE4) $=2\lceil\lg n\rceil-3$. By Cases $1,3,4,7$, and 8 , when $21 \leq$ $n \leq 28,47 \leq n \leq 64$, or $7 \times 2^{t-3}-9 \leq n \leq 2^{t}$, where $t \geq 7$, we have $d(W E 4)=2\lceil\lg n\rceil-4$. By Case 6 , when $t=6,33 \leq n \leq 46$, we have $d(W E 4)=$ $2\lceil\lg n\rceil-5$. By Cases 5 and 6 , when $37 \times 2^{t-6}-9 \leq$ $n \leq 7 \times 2^{t-3}-10$, where $t \geq 7$, we have $d(W E 4)=$ $2\lceil\lg n\rceil-5$. Finally, by Cases 9 and 10 , when $2^{t-1}$ $<n \leq 37 \times 2^{t-6}-10$, where $t \geq 8$, we have $d(W E 4)$ $=2\lceil\lg n\rceil-6$.

As already mentioned, the fan-out of $Q$ is at most 4 and the fan-out of $W V(5)$ is 4 . By Theorem 8 , the fan-out of $W V(t)$ is $3, t \geq 6$. By Property 4 and Theorem 7, the fan-out of $W(4)$ and $W L$ is 4, Hence, $W E 4(n)$ has a fan-out of 4.

Q.E.D.

\section{Comparisons of optimal prefix circuits}

For easy and exact comparisons, Table 1 gives the numbers of inputs that can be processed by representative optimal parallel prefix circuits with specific depths. From Table 1, for $n \geq 21, d(W E 4) \leq$ $d(H 4)$; that is, WE4 is better than $H 4$.

Also from Table 1, when $n=29,65 \leq n \leq 67,139$ $\leq n \leq 145,287 \leq n \leq 303,583 \leq n \leq 621,1175 \leq n$ $\leq 1259,2359 \leq n \leq 2537$, or $4727 \leq n \leq 5095$, we get $d(Z 4)=d(W E 4)-1$; however, when $45 \leq n \leq 46$, $99 \leq n \leq 102,209 \leq n \leq 214,431 \leq n \leq 438,877 \leq$ $n \leq 886,1771 \leq n \leq 1782$, or $3561 \leq n \leq 3574$, we have $d(W E 4)=d(Z 4)-1$; otherwise, $d(W E 4)=$ $d(\mathrm{Z} 4)$.

We now compare WE4 with optimal prefix circuits with unbounded fan-out. It can be checked that $d(W E 4) \leq d(M)$ except when $n=29$. As for $L Y D$, when $21 \leq n \leq 77, d(L Y D) \leq d(W E 4)$; when $78 \leq n \leq 446, d(W E 4) \leq d(L Y D)$; when $n \geq 447$, $d(W E 4)<d(L Y D)$. That is, although both $M$ and $L Y D$ have unbounded fan-out, only when $n$ is small, $d(M)$ and $d(L Y D)$ have some chances to be smaller than $d(W E 4)$ by 1 . Specifically, when $n>29, M$ can be replaced by WE4; when $n>77, L Y D$ can be replaced by $W E 4$. 
Table 1. The numbers of inputs that some optimal parallel prefix circuits with specific depths can process.

\begin{tabular}{cccc}
\hline Depth & WE4 & Z4 & H4 \\
\hline 6 & $21-28$ & $19-29$ & $19-26$ \\
\hline 7 & $29-46$ & $30-44$ & $27-41$ \\
\hline 8 & $47-64$ & $45-67$ & $42-57$ \\
\hline 9 & $65-102$ & $68-98$ & $58-87$ \\
\hline 10 & $103-138$ & $99-145$ & $88-119$ \\
\hline 11 & $139-214$ & $146-208$ & $120-179$ \\
\hline 12 & $215-286$ & $209-303$ & $180-243$ \\
\hline 13 & $287-438$ & $304-430$ & $244-363$ \\
\hline 14 & $439-582$ & $431-621$ & $364-491$ \\
\hline 15 & $583-886$ & $622-876$ & $492-731$ \\
\hline 16 & $887-1174$ & $877-1259$ & $732-987$ \\
\hline 17 & $1175-1782$ & $1260-1770$ & $988-1467$ \\
\hline 18 & $1783-2358$ & $1771-2537$ & $1468-1979$ \\
\hline 19 & $2359-3574$ & $2538-3560$ & $1980-2939$ \\
\hline 20 & $3575-4726$ & $3561-5095$ & $2940-3963$ \\
\hline
\end{tabular}

\section{Conclusion}

In this paper, we have presented Algorithm A and Algorithm B for constructing size optimal prefix circuits $W L$ and $W V$; together with $Q$ and $W$, theses circuits can be used to compose depth-size optimal parallel prefix circuit WE4. With our new approach, tedious and lengthy proofs are not required.

$W E 4$ can replace all the other optimal parallel prefix circuits with fan-out 4 except for $Z 4$. We have seen that $d(W E 4)$ may be greater than $d(Z 4)$ by 1 , $d(W E 4)$ may be less than $d(Z 4)$ by 1 , and in most cases of $n, d(W E 4)=d(Z 4)$.

\section{Acknowledgment}

This research was supported in part by the National Science Council of the R.O.C. under contract NCS 89-2213-E-011-099.

\section{References}

[1] S.G. Akl, Parallel Computation: Models and Methods, Prentice-Hall, Upper Saddle River, NJ, 1997.

[2] A. Bilgory and D.D. Gajski, "A heuristic for suffix solutions," IEEE Trans. Comput., vol. C-35, Jan. 1986, pp. 34-42.

[3] G.E. Blelloch, "Scans as primitive operations," IEEE Trans. Comput., vol. 38, Nov. 1989, pp. 1526-1538.

[4] R.P. Brent and H.T. Kung, "A regular layout for parallel adders," IEEE Trans. Comput., vol. C-31, Mar. 1982, pp. 260-264.

[5] D.A. Carlson and B. Sugla, "Limited width parallel prefix circuits," J. Supercomput., vol. 4, June 1990, pp. 107-129.

[6] J.-N. Chen, Constructing Depth-Size Opimal Parallel Prefix Circuit Z4, Master Thesis, Dept. of Electronic Engineering, National Taiwan University of Science and Technology, 2001.

[7] C.P. Kruskal, L. Rudolph, and M. Snir, "The power of parallel prefix," IEEE Trans. Comput., vol. C-34, Oct. 1985, pp. 965-968.

[8] R.E. Ladner and M.J. Fischer, "Parallel prefix computation," J. ACM, vol. 27, Oct. 1980, pp. 831838.

[9] S. Lakshmivarahan and S.K. Dhall, Parallel Computing Using the Prefix Problem, Oxford University Press, Oxford, UK,1994.

[10] Y.-C. Lin, "Optimal parallel prefix circuits with fan-out 2 and corresponding parallel algorithms," Neural, Parallel \& Scientific Computations, vol. 7, Mar. 1999, pp. 33-42.

[11] Y.-C. Lin, Y.-H. Hsu, and C.-K. Liu, "Depth-size optimal parallel prefix circuits with fan-out 4 and small depth," in Proc. Int. Conf. on Parallel and Distributed Computing, Applications, and Technologies, 2001, pp. 74-82.

[12] Y.-C. Lin and C.-K. Liu, "Constructing optimal parallel prefix circuits," in Proc. National Computer Symp., 1999, pp. C-313-320.

[13] Y.-C. Lin and C.-K. Liu, "Finding optimal parallel prefix circuits with fan-out 2 in constant time," Inform. Process. Lett., vol. 70, May 1999, pp. 191-195.

[14] Y.-C. Lin and C.-C. Shih, "Optimal parallel prefix circuits with fan-out at most 4," in Proc. 2nd IASTED Int. Conf. on Parallel and Distributed Computing and Networks, 1998, pp. 312-317.

[15] Y.-C. Lin and C.-C. Shih, "A new class of depth-size optimal parallel prefix circuits," J. Supercomput., vol. 14, July 1999, pp. 39-52.

[16] M. Snir, "Depth-size trade-offs for parallel prefix computation," J. Algorithms, vol. 7, 1986, pp. 185-201.

[17] H. Wang, A. Nicolau, and K.S. Siu, "The strict time lower bound and optimal schedules for parallel prefix with resource constraints," IEEE Trans. Comput., vol. 45, Nov. 1996, pp. 1257-1271.

[18] N.H.E. Weste and K. Eshraghian, Principles of CMOS VLSI Design: A System Perspective, 2nd ed., Addison-Wesley, Reading, MA, 1993. 